\title{
INCONTINENCIA URINARIA DE ESFUERZO ASOCIADA A OBSTRUCCIÓN DEL TRACTO URINARIO INFERIOR EN EL CISTOCELE.
}

\author{
Jesús Salinas, Sara Prieto, José María Adot, Miguel Virseda y Ángel Silmi.
}

Servicio de Urología. Hospital Clínico San Carlos. Universidad Complutense. Madrid. España.

\begin{abstract}
Resumen.- OBJETIVO: La asociación de incontinencia urinaria de esfuerzo por hipermovilidad uretral y obstrucción del tracto urinario inferior en un mismo paciente con cistocele, es rara, e incluso presenta una apariencia contradictoria. El objetivo de nuestro trabajo consistiría en tratar de definir las características que permitieran identificar a esta entidad, frente a la incontinencia urinaria de esfuerzo y obstrucción del tracto urinario inferior aisladas, en pacientes con cistocele.
\end{abstract}

MÉTODOS: Se realizó un estudio retrospectivo en 1.116 de casos de cistocele sometidos a investigación urodinámica. Todas las pacientes fueron sometidas a historia clínica, exploración física neurourológica y uroginecológica. El estudio urodinámico consistió en una flujometría, cistomanometría, test presión detrusor/flujo miccional y cistouretrografías. Se recogieron los datos en el programa Excel 2000 y el tratamiento estadístico se realizó con el paquete SPSS.

RESULTADOS: A - Datos generales: Se clasificaron para este estudio en el grupo I lincontinencia de es-

Jesús Salinas Casado

Servicio de Urología Hospital Clínico San Carlos

Martín Lagos, s/n 28040 Madrid. (España). salinascasado@yahoo.es

Trabajo recibido: 7 de febrero 2007. fuerzo aislada por hipermovilidad uretrall 25 casos; en el grupo II (obstrucción tracto urinario inferior) 24 casos; y en el grupo III (incontinencia urinaria esfuerzo asociada a obstrucción tracto urinario inferior) 14 casos. La proporción de los tres grupos en la totalidad de los cistoceles, correspondió a una relación 4/1/0.05 para los grupos H-H-III respectivamente. La edad media correspondió a 58.4 años para grupo 1, 68.2 para grupo II y 71.2 para el grupo III. Se demostró una diferencia significativa de menor edad para el grupo I ( $p<0.0005)$. B - El síntoma de sensación de bulto en vagina fue menor en el grupo / (32 \%); se demostró una diferencia significativa de $(p=0.02) ; C-E l$ incremento de la frecuencia miccional diurna fue menor en el grupo I (32\%) p=0.02. D - La frecuencia miccional nocturna fue menor en el grupo I ( 1 episodio) (p<0.004). E - El síntoma de incontinencia urinaria a la tos fue más frecuente en el grupo I (84 \%) ( $p=0.0004)$. F - Fue mayor en el grupo I la capacidad vesical $(243.6$ ml.) (p<0.05). G - El URA fue menor en el grupo I, con un valor de $37.9 \mathrm{~cm}$. H2O (p=0.01). H - El W8O-W2O fue mayor en el grupo l: $1.3 \mathrm{~W} / \mathrm{m} 2 \mathrm{p}<0.05$. y mayor en el grupo II (70.8\%) (p=0.02). I - El grado radiológico de cistocele fue mayor en el grupo II (1.7) (p<0.05). $\mathrm{J}$ - La hiperactividad del detrusor fue más frecuente en el grupo III (64.3\%) (p=0.00009). K - No se demostraron diferencias significativas en el tipo de obstrucción entre los grupos II-II.

CONCLUSIONES: El grupo de incontinencia urinaria de esfuerzo aislada (grupo II se caracterizaría por una menor edad, con una menor frecuencia de sensación de bulto en vagina, menor frecuencia miccional diurna y nocturna y unos datos urodinámicos de mayor capacidad vesical, menor resistencia uretral y contractilidad normal del detrusor. El grupo de obstrucción aislada del tracto urinario inferior (grupo III) se caracterizaría por una mayor frecuencia de bulto en vagina, e incremento del grado de cistocele radiológico. El grupo de incontinencia urinaria de esfuerzo asociada a obstrucción del 
tracto urinario inferior, se acompañó de un mayor porcentaje de casos de hiperactividad del detrusor. Todos estos datos podrían permitir la correcta identificación de los diferentes elementos de riesgo, en los distintos grupos.

Palabras clave: Incontinencia urinaria de esfuerzo. Obstrucción tracto urinario inferior. Cistocele.

Summary.- OBJECTIVES: The association of stress urinary incontinence secondary to urethral hypermobility and lower urinary tract obstruction in the same patient with cystocele is rare, and even represents a contradiction. The objective of our work is to treat to define the characteristics that identify this entity, in comparison with isolated stress urinary incontinence or lower urinary tract obstruction in patients with cystocele.

METHODS: We performed a retrospective study in 1168 cases of cystocele in which urodynamic studies were performed. All patients underwent history and neurological and uro-gynecologycal physical examination. The urodynamic study included uroflowmetry, cystomanometry, voiding pressure/flow tests and voiding cystourethrograms. All data were collected in an Excel 2000 database and statistical analysis was performed with the SPSS software.

RESULTS: A- General data: 25 cases qualified for the study in group I (isolated stress urinary incontinence with urethral hypermobility); 24 cases in group II llower urinary tract obstruction); and 14 cases in group III (astress urinary incontinence associated with lower urinary tract obstruction). The proportion of each group in the whole group of cystoceles corresponded to a 4/1/0.05 ratio respectively. Mean age was 58.4 years for group I, 68.2 for group II and 71.2 for group III. A Statistically significant lower age was demonstrated for group I / $p$ $<0.0005)$. B-The symptom "sensation of vaginal lump" was less frequent in group / (32\%). A significant difference was demonstrated ( $p=0.02$ ). C-Group / showed a lower increase of daily voiding frequency $(32 \%), p=$ 0.02. D-Group I showed less night-ime voiding frequency $(1$ episode) $(p<0.04)$. E-Urinary incontinence with cough was less frequent in group I (84\%) ( $p=0.0004)$. F- Group I had more bladder capacity $(243.6 \mathrm{ml})$ (p < 0.05). G-Group I showed less urethral resistances (URA $=37.9 \mathrm{~cm} \mathrm{H} 2 \mathrm{O})(\mathrm{p}=0.011 . \mathrm{H}-\mathrm{W} 80-\mathrm{W} 20$ was higher in group I: $1.3 \mathrm{~W} / \mathrm{m} 2(p<0.05)$. I-The symptom "sensation of vaginal lump appeared more often in group II (70.8\%) (p = 0.02). J-Radiological degree of cystocele was greater in group II (1.7) ( $p<0.05)$. K- Detrusor hyperactivity was more frequent in group III (64.3\%) $p=$ 0.00009). L- No significant differences were found between groups II-III when comparing type of obstruction.
CONCLUSIONS: The group of isolated stress urinary incontinence (group II) is characterized by a younger age, less frequency of sensation of vaginal lump, less daily frequency and nocturia, and urodynamic data of greater bladder capacity, lower urethral resistance and normal detrusor contractility. The group of isolated lower urinary tract obstruction (group II) could be characterized by a more frequent sensation of vaginal lump and increase of the radiological cystocele. The group of stress urinary incontinence associated with lower urinary tract obstruction had a higher percentage of cases of detrusor hyperactivity. All these data might enable a proper identification of different risk elements in the groups.

Keywords: Stress urinary incontinence. Lower urinary tract obstruction. Cystocele.

\section{INTRODUCCIÓN}

La presencia de cistocele y la concomitancia de incontinencia urinaria de esfuerzo ocurren frecuentemente dentro del fenómeno global de la relajación del suelo pélvico, (1-4).

Sin embargo Adot y cols. (5), aunque también demuestra aquellos datos, no encuentra relación estadísticamente significativamente entre la presencia y grado de cistocele en una parte, e incontinencia urinaria de esfuerzo por otra parte.

En otro sentido, es bien conocido que la distorsión uretral debida a prolapso del compartimiento anterior, puede causar obstrucción del tracto urinario inferior (6).

En esta línea, Salinas y cols. (7) tampoco encontraron relación significativa entre la presencia y grado de cistocele frente a la obstrucción del tracto urinario inferior.

La asociación de incontinencia urinaria de esfuerzo por hipermovilidad uretral y obstrucción del tracto urinario inferior en un mismo paciente con prolapso del compartimiento anterior, es rara, e incluso presenta una apariencia contradictoria, no habiendo sido descrita hasta la fecha.

El objetivo de nuestro trabajo consistiría en tratar de definir las características que permitieran identificar a esta nueva entidad, con relación estadísticamente significativa, frente a la incontinencia urinaria de esfuerzo y obstrucción del tracto urinario inferior aisladas, en pacientes con cistocele. 


\section{MATERIAL Y MÉTODOS}

Se realizó un estudio retrospectivo en el período comprendido entre enero 2003 y junio 2006, en los 1.116 casos de cistocele, remitidos a la Unidad de Urología Funcional, para estudio urodinámi$\mathrm{co}$, atendiendo al diagnóstico de incontinencia urinaria de esfuerzo aislada por hipermovilidad uretral (grupo I), obstrucción tracto urinario inferior (grupo II), incontinencia urinaria de esfuerzo asociada a obstrucción tracto urinario inferior (grupo III). Todos las pacientes fueron sometidas a historia clínica, exploración física neurourológica y uroginecológica.

El estudio urodinámico consistió en una flujometría, cistomanometría, test presión detrusor/flujo miccional. El estudio radiológico se refirió a las cistouretrografías.

El flujo miccional, además de valorar los parámetros de flujo máximo y medio, se interpretó mediante el nomograma de Haylen para la mujer adulta, que clasifica con los valores en percentiles. Se consideró patológico la existencia de un residuo postmiccional mayor del $20 \%$ del volumen miccional.

El grupo de cistocele clínico se consideró Grado 0 (no cistocele), ante la ausencia de prolapso vesical durante la tos. Grado 1, ante descenso leve de la misma; Grado 2 cuando se produce descenso, con la tos o el valsalva, hasta el introito vaginal y Grado 3, cuando desciende por debajo del introito vaginal. Se excluyeron los casos con otros prolapsos pélvicos, antecedentes de cirugía del suelo pélvico y patología neurológica. La resistencia uretral se midió mediante el URA (resistencia uretral media). (cm. $\mathrm{H}_{2} \mathrm{O}$.

La tipificación de la obstrucción estructural se realizó mediante el PURR (relación de resistencia uretral pasiva) (calsificación Chess), en compresiva y constrictiva (grado 1, 2 y 3). La potencia contráctil del detrusor se valoró mediante el Wmax (parámetro de contractilidad isométrica) y $W_{80}-W_{20}$ (parámetro de contractilidad isotónica). La terminología urodinámica y medidas siguieron las normas de la Internacional Continente Society, ICS.

El estudio radiológico (cistouretrográfias), valoró el grado de cistocele respecto a la sínfisis del pubis $(0,1,2,3)$, morfología vesical y competencia cuello en el llenado vesical, así como las alteraciones de la morfología uretral en la micción.

Se recogieron los datos en el programa Excel 2000 y el tratamiento estadístico se realizó con el paquete SPSS.
La probabilidad de los diferentes análisis estadísticos se consideró significativa por debajo de 0.05 , analizándose las variables cuantitativas mediante el test de la $t$ de Student, y las variables no paramétricas mediante la $\mathrm{CHi}$ cuadro de Pearson.

\section{RESULTADOS}

\section{A - Datos generales}

Se clasificaron para este estudio en el grupo I (incontinencia urinaria de esfuerzo aislada por hipermovilidad uretral) 25 casos; en el grupo II (obstrucción tracto urinario inferior) 24 casos, y en el grupo III (incontinencia urinaria esfuerzo asociada a obstrucción tracto urinario inferior) 14 casos. La proporción de los 3 grupos en la totalidad de los cistoceles, correspondió a una relación 4/1/0.05 para los grupos I,II y III respectivamente.

La edad media correspondió a 58.4 años para el grupo I (Stress aislado) 68.2 para el grupo II (obstrucción) y 71.2 años para el grupo III (stress asociado a obstrucción). Se demostró una diferencia significativa entre stress (grupol) y obstrucción (grupo II) ( $p=0.003)$, y entre stress (grupo l) y stress asociado a obstrucción (grupo III) ( $p=0.002$ )

\section{B - Datos clínicos}

- Incontinencia a la tos; 84 \% (grupo I). $28.6 \%$ (grupo II). $28.6 \%$ (grupo III). Se demostraron diferencias significativas ( $p=0.0004)$.

- Frecuencia miccional diurna aumentada: 32 \% (grupo I). $62.5 \%$ (grupo II). $71.4 \%$ (grupo III). Se demostraron diferencias significativas entre los grupos ( $p=0.02)$.

- Frecuencia miccional nocturna: 1 episodio (grupo I) 2.4 episodios (grupo II). 2.4 episodios (grupo III). Se demostraron diferencias significativas entre grupo I y II ( $p=0.003)$, y entre grupo I y III $(p=0.002)$.

- Sensación de bulto en vagina: $32 \%$ (grupo I). 70.8 $\%$ (grupo II) y $57 \%$ (grupo III). Se demostraron diferencias significativas entre los grupos $(p=0.02)$.

No se demostró diferencias significativas en el porcentaje de retención aguda de orina $(p=0.16)$, urgencia miccional $(p=0.07)$, urgencia-incontinencia $(p=0.07)$, dificultad miccional $(p=0.91)$, alteraciones en el calibre miccional $(p=0.29)$, número de compresas utilizadas $(p=0.96)$, y relación con la edad $(p=0.81)$.

\section{C - Datos exploración física}

No se demostraron diferencias en el grado de cistocele $(p=0.59)$.

\section{D - Datos flujometría}

El flujo miccional máximo fue de $19.3 \mathrm{ml} / \mathrm{s}$ 
TABLA I. DATOS DIFERENCIALES CLÍNICOS GRUPO I, II, III. SBU: SENSACIÓN DE BULTO EN VAGINA. IFMD: INCREMENTO FRECUENCIA MICCIONAL DIURNA. FMN: FRECUENCIA MICCIONAL NOCTURNA ICT: INCONTINENCIA TOS.

\begin{tabular}{|c|c|c|c|c|c|c|c|c|c|}
\hline & $\begin{array}{l}\text { EDAD } \\
\text { (años) }\end{array}$ & & $\begin{array}{c}\text { SBV } \\
\text { (si/no) }\end{array}$ & & $\begin{array}{l}\text { IFMD } \\
\text { (si/no) }\end{array}$ & & odios) & & $\begin{array}{c}\text { ICT } \\
\text { (si/no) }\end{array}$ \\
\hline III & 71 & III & $57 \%$ & III & $57 \%$ & & 2 & III & $29 \%$ \\
\hline$\overline{\text { III }}$ & 68 & $\|$ & $71 \%$ & II & $70 \%$ & $\|$ & 2 & $\|$ & $29 \%$ \\
\hline 1 & 58 & & $32 \%$ & 1 & $32 \%$ & 1 & 1 & 1 & $84 \%$ \\
\hline
\end{tabular}

para el grupo I, $9.9 \mathrm{ml} / \mathrm{s}$ grupo ll y 15 para el grupo III. Se demostraron diferencias significativas entre el grupo I y III $(p=0.000)$. El percentil del flujo máximo fue de 42 (grupo I), 12.2 (grupo II) y 27.3 (grupo III). Se demostraron diferencias significativas entre grupo | y || $(p=0.00)$.

El flujo medio fue de $8.1 \mathrm{ml} / \mathrm{s}$ para el (grupo I), 4.7 (grupo II) y 7 (grupo III). Se demostraron diferencias significativas entre grupo I y ||$(p=0.000)$. El percentil del flujo medio fue de 26.6 (grupo l), 8 (grupo II) y 23.4 (grupo III). Se demostraron diferencias significativas entre grupo I y II $(p=0.04)$.

No se demostraron diferencia significativas en el volumen miccional $(p=0.18)$, residuo postmiccional $(p=0.08)$, y morfología de la curva $(p=0.28)$.

\section{E - Datos cistomanometría}

Se demostró una capacidad vesical de 243.6 $\mathrm{ml}$ en el grupo I, 200.6 en el grupo II y 181.6 en el grupo III. Se demostraron diferencias significativas entre el grupo I y III ( $p=0.02)$. La primera sensación vesical fue de $164 \mathrm{ml}$ en el grupo I, 122.8 en el grupo II, y 116.8 en el grupo III. Se demostraron di- ferencias significativas entre el grupo I y III $(p=0.01)$, y entre grupo I y II $(p=0.03)$.

No se demostraron diferencias significativas en la presión de llenado ( $p=0.55)$.

La hiperactividad del detrusor se demostró en el $8.3 \%$ en el grupo I, $45.8 \%$ en el grupo II y $64.3 \%$ en el grupo III. Se comprobó una diferencia significativa entre los grupos $(p=0.0009)$. No se demostraron diferencias significativas en la amplitud $(p=0.16)$ y capacidad vesical $(p=0.17)$ de las contracciones involuntarias del detrusor.

\section{F - Datos presión detrusor/flujomiccional}

La presión detrusor máxima miccional fue de $37.9 \mathrm{~cm}$. $\mathrm{H} 2 \mathrm{O}$ para el grupo I, $68.4 \mathrm{~cm}$. $\mathrm{H}_{2} \mathrm{O}$ para el grupo II y $59.4 \mathrm{~cm}$. $\mathrm{H}_{2} \mathrm{O}$ para el grupo III. Se demostraron diferencias significativas entre el grupo I y II $(p=0.000)$, y entre el grupo I y III $(p=0.01)$.

EI URA (parámetro de resistencia uretral) fue de 10.4 para el grupo I, 39.7 para el grupo II y 38.4 para el grupo III. Se demostraron diferencias significativas entre el grupo I y II $(p=0.000)$ y grupo I y III $(p=0.000)$.

TABLA II. DATOS DIFERENCIALES URODINÁMICOS Y RADIOLÓGICOS GRUPO I, II, III. HD: HIPERACTIVIDAD DETRUSOR. GCR: GRADO CISTOCELE RADIOLÓGICO.

\begin{tabular}{|c|c|c|c|c|c|c|c|c|c|}
\hline \multicolumn{2}{|r|}{$\begin{array}{l}\text { CAPACIDAD } \\
\text { VESICAL (ml) }\end{array}$} & \multicolumn{2}{|r|}{$\begin{array}{c}\text { URA } \\
\text { (cm H2O) }\end{array}$} & \multicolumn{2}{|r|}{$\begin{array}{c}\text { HD } \\
\text { (si/no) }\end{array}$} & \multicolumn{2}{|c|}{$\begin{array}{l}\text { W80-W20 } \\
(\mathrm{w} / \mathrm{m} 2)\end{array}$} & \multicolumn{2}{|c|}{$\begin{array}{c}\text { GCR } \\
\text { (grados) }\end{array}$} \\
\hline III & 181 & III & 38 & III & $64 \%$ & & -0.1 & III & 2 \\
\hline II & 200 & $\|$ & 40 & II & $46 \%$ & II & -0.3 & II & 2 \\
\hline 1 & 243 & 1 & 10 & & $8 \%$ & & +1 & 1 & 1 \\
\hline
\end{tabular}


En relación al tipo de obstrucción constrictiva, compresiva, mixta, (compresiva y constrictiva), se demostraron en el grupo II: constrictiva $67 \%$, compresión $8 \%$, mixta $16 \%$, no obstrucción $4 \%$, no valorable $4 \%$. Se demostraron en el grupo III: constrictiva $85 \%$, compresiva $0 \%$, mixta $7 \%$, y no obstrucción $7 \%$. No se demostraron diferencias significativas entre el grupo II y III, en la presencia de un componente obstructivo constrictivo y un componente obstructivo compresivo $(p=0.16)$. Aunque no se demostraron diferencias significativas $(p=0.61)$ en la presencia de hiperactividad detrusor en la obstrucción compresiva/constrictiva, sí las hubo en la amplitud $61.5 \mathrm{~cm} . \mathrm{H}_{2} \mathrm{O}$ en la constrictiva versus 104 en las compresivas ( $p=0.007$ ), y capacidad vesical de las contracciones: $137 \mathrm{ml}$ en las constrictivas versus 58 $\mathrm{ml}$ en las compresivas ( $\mathrm{p}=0.003)$.

La contractilidad isotónica del detrusor medida por el $\mathrm{W}_{80}-\mathrm{W}_{20}$, demostró un valor de $1.3 \mathrm{~W} / \mathrm{m}^{2}$ para el grupo l, -0.35 para el grupo Il y -0.13 para el grupo III. Se comprobaron diferencias significativas entre el grupo I y II $(p=0.02)$ y entre el grupo I y III $(p=0.04)$. No se demostraron diferencias significativas en el parámetro de contractilidad isométrica del detrusor, $W \max (p=0.24)$.

\section{G - Datos cistouretrográficos}

Se demostró un grado de cistocele 1.1 en el Grupo I, 1.7 en el Grupo II, y 1.6 en el Grupo III. Se demostraron diferencias significativas entre el Grupo I y II ( $p=0.02)$, y tendencia hacia la significación entre el Grupo I y III ( $p=0.05)$. El grado de incremento del cistocele con el valsalva no presentó diferencias significativas $(p=0.33)$. No se observaron asímismo diferencias significativas en la morfología vesical $(p=0.24)$, competencia cuello vesical $(p=0.8)$, y alteraciones morfológicas uretral miccionales (distorsión, dilatación ...) ( $p=0.49)$.

En las Tablas I y II se recogen, los datos diagnósticos, fundamentales, tanto clínicos y urodinámicos, como radiológicos, en los diferentes grupos.

\section{DISCUSIÓN}

Es de señalar en la serie general la mayor asociación del cistocele con la incontinencia urinaria de esfuerzo, frente a la asociación del cistocele con la obstrucción del tracto urinario inferior. La asociación de la incontinencia urinaria de esfuerzo y obstrucción del tracto urinario inferior en las pacientes con cistocele es muy rara.

No hemos sido capaces de demostrar la patofisiología de las pacientes del Grupo III, ya que aunque se comprobó incrementado el parámetro de resistencia uretral correspondiente a la existencia de obstrucción, no se encontraron diferencias significativas, ni en el valor del URA, ni en el tipo de obstrucción (componente compresivo/constrictivo) que le permitiera distinguir del Grupo II.

Esto es, en los dos Grupos II y III, fue muy frecuente la obstrucción constrictiva, tal como describieron Salinas y Valentíni y cols. (7 y 8), en los cistoceles. Esto significa que la disminución del área de sección uretral durante la micción por mecanismos no bien objetivados, aumentaría la resistencia al flujo y sería la causa que explicaría este tipo de obtención. Fue más rara la obstrucción compresiva en la que se produce un aumento de la presión de apertura vesical por una causa extrínseca que actúe estrechando sobre cuello vesical y uretra.

Estas consideraciones que son sobre todo aplicables al varón adulto (9), tampoco se demuestran inequívocamente en éstos, cuando se compara la hiperplasia benigna de próstata con la obstrucción compresiva; la estenosis uretral con la obstrucción constrictiva y la estenosis cuello vesical con la obstrucción mixta (compresiva y constrictiva).

El estudio radiológico convencional (cistouretrografías) no apuntó datos morfológicos que ayudaran al diagnóstico diferencial entre los grupos II y III. Hoy en día, se están aplicando técnicas de imagen dinámicas como la ecografía introital, resonancia magnética del suelo pélvico, colpocistodefecografía, Halligan y cols. (10) que están demostrando su utilidad, así como técnicas tridimensionales tanto ecográficas, Weinstein y cols. (11) como de RMN, Otcenasek(12), pero son insuficientes para valorar la dinámica de los distintos compartimentos del suelo pélvico, no sólo durante el llenado vesical, sino durante la micción.

De esta forma, podríamos llegar a entender porqué un cistocele no sólo produce incontinencia urinaria de esfuerzo aislada, sino porque en otros casos obstrucción en la micción, y en otros, incontinencia durante el llenado vesical, así como obstrucción durante la micción.

Algunos autores, tratan de contestar a estas preguntas. Así, Porena y cols. (13), tratan de explicar la obstrucción en algunos casos por una caída del retrotrígono por debajo de la uretra. Dietz y cols. (14), comprueba ecográficamente que aparte del prolapso del compartimiento anterior, el enterocele también puede afectar negativamente al flujo miccional de estas pacientes. Contrariamente a lo que en un principio parece- 
ría, el prolapso uterino no altera significativamente la micción.

La urodinámica-resonancia de Borghesi y cols. (15) podría aportar nuevos datos en el estudio de la patofisiología de las disfunciones del suelo pélvico en la mujer, y es posible que la aplicación de todas estas nuevas tecnologías puedan llenar los vacíos que actualmente existen en la comprehensión de estos fenómenos.

La incontinencia urinaria de esfuerzo aislada en nuestra serie se presentó en las pacientes más jóvenes, frente a la obstrucción y stress más obstrucción, que se incrementa con la edad.

El síntoma de sensación de bulto en vagina fue así menos frecuente en las pacientes del Grupo I, frente a las del Grupo II, donde fue más frecuente. Así mismo, el dato del síntoma sensación de bulto en vagina, se correlacionó en esta serie con un mayor grado de cistocele radiológico. Esta correlación no suele ser frecuente dada la subjetividad de la exploración física (con su variación interindividual), así como la variabilidad de las diferentes técnicas diagnósticas.

La menor frecuencia miccional diurna y nocturna en el Grupo I podría estar en relación con incremento de la capacidad vesical y la ausencia de hiperactividad del detrusor.

La hiperactividad del detrusor fue más frecuente en el Grupo III, posiblemente en relación a la suma de la hiperactividad desencadenada por la incontinencia urinaria de esfuerzo y la hiperactividad asociada a la obstrucción del tracto urinario inferior. La mayor capacidad vesical, demostrada cistomanométricamente, en las pacientes del Grupo I, podría explicarse por la ausencia de contracciones involuntarias del detrusor durante el llenado vesical, frente a la mayor frecuencia de hiperactividad del detrusor, en el Grupo III.

Las pacientes con incontinencia urinaria de esfuerzo aislada (Grupo I), con una patofisiología clara, presentaron la menor resistencia uretral (medida con el URA), así como una contractilidad isotónica del detrusor, (medida por el $\mathrm{W}_{80}-\mathrm{W}_{20}$ ) dentro de la normalidad. Contrariamente las pacientes del Grupo II y III, presentaron una resistencia uretral aumentada, y una afectación de la contractilidad isotónica del detrusor. Este último dato no es infrecuente, y podría estar en relación con las obstrucciones de larga evolución.

\section{BIBLIOGRAFÍA y LECTURAS RECOMENDADAS ( ${ }^{*}$ lectura de interés $y^{* *}$ lectura fundamental)}

1. GRODY, M.H.: "Urinary incontinencia and concomitant prolapse”. Clin. Obstet. Gynecol., 41: 777, 1998.

2. COSIMO, O.; PIERLUIGI, P.; ANGELO, Z. y cols.: "A clinical and urodynamic study of patients with varying degrees of cistocele”. Maturitas, 27: 125, 1997.

3. GARDY, M.; KOZMINSKI, M.; DELANCEY, J. y cols.: "Stress incontinence and cystoceles". J. Urol., 145: 1211, 1991.

4. ROMANZI, L.; CHAIKIN, D.; BLAIVAS, J.: "The effect of genital prolapse on voiding". J. Urol., 161: $581,1999$.

*5. ADOT, J.M.; SALINAS, J.; DAMBROS, M. y cols.: "Alteraciones de la fase de llenado vesical y cistocele". Arch. Esp. Urol., 58: 309, 2005.

6. RICHARDSON, D.A.; BENT, A.E.; OSTERGARD, D.R.: "The effect of uterovaginal prolapse on urethrovesical pressure dynamics". AMJ Obstet. Gynecol. 146: 901, 1983.

**7. SALINAS, J.; ADOT, J.M.; DAMBROS, M. y cols.: "Factores de descompensación miccional y cistocele". Arch. Esp. Urol., 58: 316, 2005.

*8. VALENTÍNI, F.; BESSON, G.; NELSON, P. y cols.: "A mathematical micturition model to restore simple flow recordings healthy and syntomatic individuals and enhace uroflow interpretation". Neurourol and Urodynamics, 19: 153, 2000.

9. SCHÄFER, W.: "The contribution of the bladder outlet to the relation between pressure and flow rate during micturition". Hinman F, ed. The prostate. Newyork. Springer-Verlag, 470, 1983.

10. HALliGAN, S.; SPENCE-JONES, C.; KAMM, M.A. y cols.: "Dynamic cystoproctography and physiological testing in women with urinary stress incontinence and urogenital prolapse". Clin. Radiol., 51: 785, 1996.

11. WEINSTEIN, M.; JUNG, S.; PRETORIUS, D. y cols.: "Reliable measures of the pubovisceral muscle can be obtained with 3 D ultrasound". Neurourology and urodynamics, 25: 623, 2006.

12. OTCENASEK, M.; PAN, M.; BACA, V. y cols.: "Avulsion of the puborectal muscle, an MRI based 3D reconstruction". Neurourology and Urodynamics, 25: 626, 2006.

13. PORENA, M.; BISCOTTO, S.; CONSTANTINI, E. y cols.: "Perugia urodynamic method of analysis (PUMA): A new advanced method of urodynamic analysis applied clinically and compared with other advanced methods". Neurourology and Urodynamics, 22: 206, 2003.

*14. DIETZ, H.P.; HAYLEN, B.T.; VANCAILLIE, T.G.: "Female pelvic organ prolapse and voiding function". International Urogynecology Journal, 13: 284, 2002.

15. BORGHESI, G.; SIMONETTI, R.; GOLDMAN, S. y cols.: "MRI urodynamics as a more sensitive method to detect sphincter insufficiency in patients with SUI”. Neurology and urodynamics, 25: 627, 2006. 